This is an Open Access article distributed under the terms of the Creative Commons Attribution-Noncommercial License, which permits unrestricted use, distribution, and reproduction in any noncommercial medium, provided the original work is properly cited.

\title{
COOLING LINES AS PROBES OF THE FORMATION AND BUILDUP OF GALAXIES AND BLACK HOLES
}

\author{
P.P. van der Werf $^{1}$ and M. Spaans ${ }^{2}$ \\ ${ }^{1}$ Leiden Observatory, Leiden University, P.O. Box 9513, NL - 2300 RA Leiden, The Netherlands \\ ${ }^{2}$ Kapteyn Astronomical Institute, Groningen University, P.O. Box 800, NL - 9700 AV, Groningen, The Netherlands
}

\begin{abstract}
We discuss the use of SPICA to study the cosmic history of star formation and accretion by supermassive black holes. The cooling lines, in particular the high- $J$ rotational lines of $\mathrm{CO}$, provide a clear-cut and unique diagnostic for separating the contributions of star formation and AGN accretion to the total infrared luminosity of active, gasrich galaxies. We briefly review existing efforts for studying high- $J \mathrm{CO}$ emission from galaxies at low and high redshift. We finally comment on the detectability of cooling radiation from primordial (very low metallicity) galaxies containing an accreting supermassive black hole with SPICA/SAFARI.
\end{abstract}

Key words: Galaxies: formation - Galaxies: nuclei - Missions: SPICA

\section{INTRODUCTION}

For several decades, our only direct probes of the highredshift universe were QSOs and radio galaxies. While significant insight into the properties and evolution of these populations was obtained, almost nothing was known about the population of normal galaxies at high $z$. This has changed radically over the last decade. Deep imaging and spectroscopy from the ground and from space have revealed a rich and diverse population of high- $z$ galaxies, and enormous progress has been made in establishing a complete inventory of the high- $z$ universe, in terms of mass and metallicity budget, energy output and evolution over cosmic time. In addition, first investigations of the genesis of the fundamental scaling relations of galaxies (e.g., the fundamental plane and the Tully-Fisher relation) are being undertaken, and will provide insight into the origin of the galaxy population itself.

One of the most remarkable scaling relations is the $M_{\bullet}-\sigma$ relation, i.e., the relation between the mass of a nuclear supermassive black hole (SMBH) and the velocity dispersion of the spheroid (elliptical or bulge) hosting the black hole (Magorrian et al., 1998; Ferrarese \& Merritt, 2000). The fact that the black hole mass and total galaxy mass are closely related is truly remarkable, given that there is a factor of $\sim 10^{8}$ between the AUsize Schwarzschild radius of the black hole and the kpc- size dimension of the galaxy. This is generally interpreted as evidence that the formation and growth of the SMBH is directly related to the formation process of the stellar population, e.g., in a violent burst of star formation. The picture of the simultaneous build-up of a SMBH with an extreme burst of star formation provides a new context for the luminous and ultraluminous infrared galaxies (LIRGs and ULIRGs), long suggested to be the birthplaces of SMBHs powering active galactic nuclei (AGNs). Studies of local ULIRGs demonstrate that they plausibly evolve into moderate mass field ellipticals (Genzel et al., 2001). The giant ellipticals in the local universe would then be the result of similar but much more extreme events at higher redshifts. Indeed, the population of submillimetre galaxies (SMGs) provides the more luminous high redshift counterpart of the local LIRGs and ULIRGs and may be responsible for the formation of local massive spheroids and for generating QSO activity at high redshift (Blain et al., 1999).

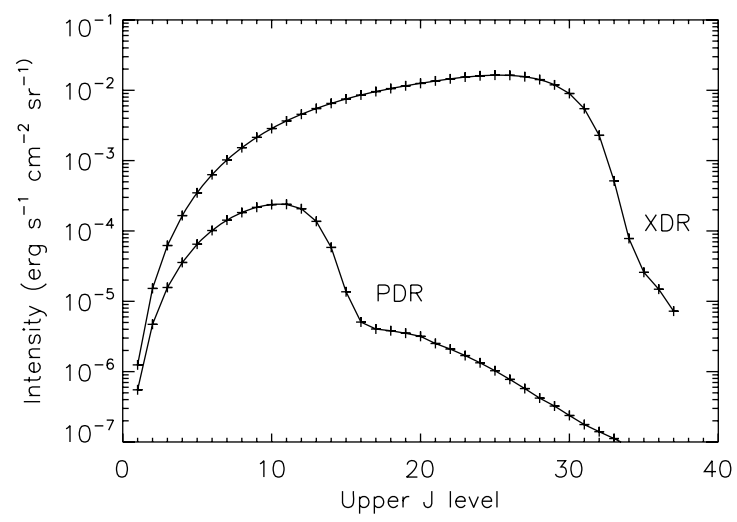

Figure 1. CO emergent intensity distributions for a PDR and an $X D R$ with identical impinging energy density, as described in Section 2. Note that high-J CO emission is expected in both cases, but vastly more prominently in the XDR (Spaans 83 Meijerink, 2008).

\section{STAR FORMATION HISTORY AND ACCRETION HISTORY}

Theoretically, a number of mechanisms have been proposed for establishing the $M_{\bullet}-\sigma$ relation (Murray et al., 
2005; Croton, 2006), but attempts at observational tests of these models are extremely challenging and have sometimes produced conflicting results (Shields et al., 2006; Alexander at al., 2005).

SPICA provides a new window on this problem with its sensitivity in the far-infrared region. This region contains the key cooling transitions of photon-dominated regions (PDRs, heated by stellar UV radiation) and X-ray dominated regions (XDRs, heated by the X-ray radiation from an accreting SMBH). Advanced PDR/XDR models (Spaans \& Meijerink, 2008) reveal the significantly different spectral patterns from XDRs and PDRs. In both cases cooling is provided by the principal fine-structure lines ([C II $158 \mu \mathrm{m}$, [O I] 63 and $145 \mu \mathrm{m}$ with in some regions of parameter space contributions from [Si II] and [Fe II] lines) as well as rotational lines of $\mathrm{CO}$ and rotational and vibrational lines of $\mathrm{H}_{2}$. However, the flux distribution over the $\mathrm{CO}$ rotational ladder is vastly different for PDRs and XDRs. In the UV, a $10 \mathrm{eV}$ photon penetrates a column of about $10^{21} \mathrm{~cm}^{-2}$ (for solar metallicity) before being absorbed by dust grains. UV photons also have the right energy to dissociate $\mathrm{CO}$ between 11.2 en $13.6 \mathrm{eV}$. In contrast, a $1 \mathrm{keV}$ X-ray photon traverses a column of $10^{22} \mathrm{~cm}^{-2}$ before absorption by hydrogen and metals occurs, since the absorption cross section scales as $\sim 1 /$ energy $^{3}$. Because of their high energy, neither X-rays nor the induced secondary electrons destroy CO efficiently. Furthermore, UV photons cause photoelectric heating when electrons are liberated from (small) dust grains, with an efficiency of no more than $1 \%$. X-rays on the other hand heat through ionization and (mostly) the subsequent Coulomb interactions of liberated very fast electrons, with an efficiency of about $10-50 \%$ (depending on the electron abundance). Consequently, PDRs, associated with star formation, contain CO layers that are relatively cool $(10-100 \mathrm{~K})$, while XDRs, associated with black hole accretion, are characterized by deeper layers of much warmer CO $(20-500 \mathrm{~K})$.

In Fig. 1 a comparison is presented between a PDR and an XDR for a density of $n=10^{5} \mathrm{~cm}^{-3}$ and an illuminating radiation field of $160 \mathrm{erg} \mathrm{s}^{-1} \mathrm{~cm}^{-2}$, representative of a region like the Orion Bar in the Milky Way (in the UV) or gas at a hundred parsecs from an accreting black hole powering a Seyfert nucleus with a luminosity of $10^{44} \mathrm{erg} \mathrm{s}^{-1}$ (in X-rays). It should be noted that the PDR case included here pushes the most extreme range expected in starburst galaxies, while X-ray fluxes as low as $5 \mathrm{erg} \mathrm{s}^{-1} \mathrm{~cm}^{-2}$, impinging on $\sim 10^{5} \mathrm{~cm}^{-3}$ gas are still capable of producing substantial $\mathrm{CO}$ emission at very high $J$ CO levels. The CO ladder at high $J$ levels thus constitutes a unique diagnostic of SMBH accretion activity in the SPICA/SAFARI wavelength range.

Real galaxies will of course produce a mixture of PDR and XDR emission. Separating these will require (a) a complete inventory of the cooling emission lines at high sensitivity; (b) accurate modeling with state-of-the-art PDR and XDR codes, including radiative transfer in 3 dimen- sions; (c) additional data for constraining local densities (density-tracing molecules), column densities (isotopomeric lines), sizes of emission regions (high resolution ALMA imaging), as well as high-quality X-ray and infrared data. All of these will be in reach within the coming decade, and thus we may have some optimism that a reliable separation of star formation and AGN as contributors to the total luminosity of galaxies (which has been elusive problem for many years) can indeed be achieved with SPICA.

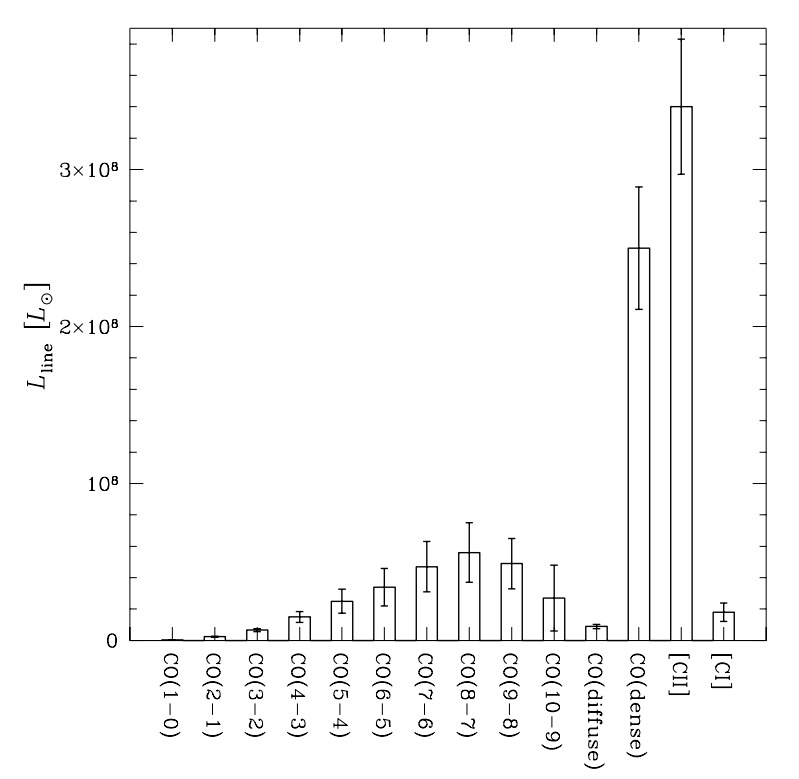

Figure 2. Cooling budget in Mrk231 (Papadopoulos et al., 2007), based on observations until $C O J=6-5$ and a wellconstrained model for the higher lines. The CO is modeled as a combination of a diffuse and a dense phase, with the dense phase containing most of the mass and emitting most of the cooling radiation, comparable in total luminosity to the [C II] line.

\section{StATE-OF-THE-ART: LOW- $z$ GALAXIES}

Reasonably detailed CO spectral line energy distributions are gradually becoming available, for instance for M82 (Mao et al., 2000) and NGC 253 (Bradford et al, 2003; Güsten et al., 2006), although for low- $z$ galaxies they still do not probe higher rotational levels than $J=7$. The first high-quality CO flux distribution for a ULIRG was presented by Papadopoulos et al. (2007) for the nearby ULIRG Mrk 231. These authors conclude that the CO ladder requires a two-component model: one component of fairly diffuse gas dominating the low- $J$ lines and one component of dense gas dominating at $J>4$. Remarkably, it was found that the dense component dominates the molecular gas mass as well as the total cooling radiation from Mrk 231. The total cooling by this component approaches that by the $[\mathrm{C}$ II] line, implying a totally different thermal 
balance than in for instance the Milky Way. The derived cooling budget is shown in Fig. 2. This result also sheds new light on the discovery with the ISO satelite that in ULIRGs the $[\mathrm{C} \mathrm{II}] / L_{\mathrm{IR}}$ ratio is about a factor of 10 lower than in lower luminosity galaxies (where the [C II] $158 \mu \mathrm{m}$ line typically contains $0.1-0.5 \%$ of $L_{\mathrm{IR}}$ ). Physically, this situation can arise in dense PDRs (where the ionized carbon layer is thin and the molecular gas layer is close to the heating source), in XDRs, or in PDRs with an enhanced cosmic ray flux. These possibilities can be distinguished using the more comprehensive data that will be obtained by the Herschel Comprehensive ULIRG Emission Survey (HerCULES). This project will obtain full spectra of the CO ladder from $J=5$ to 13 as well as the key cooling lines of [C II] $(158 \mu \mathrm{m})$ and [O I] $(63$ and $145 \mu \mathrm{m})$ for a flux-limited sample of 29 local LIRGs and ULIRGs. This project will be an important pathfinder for future studies with SPICA/SAFARI.

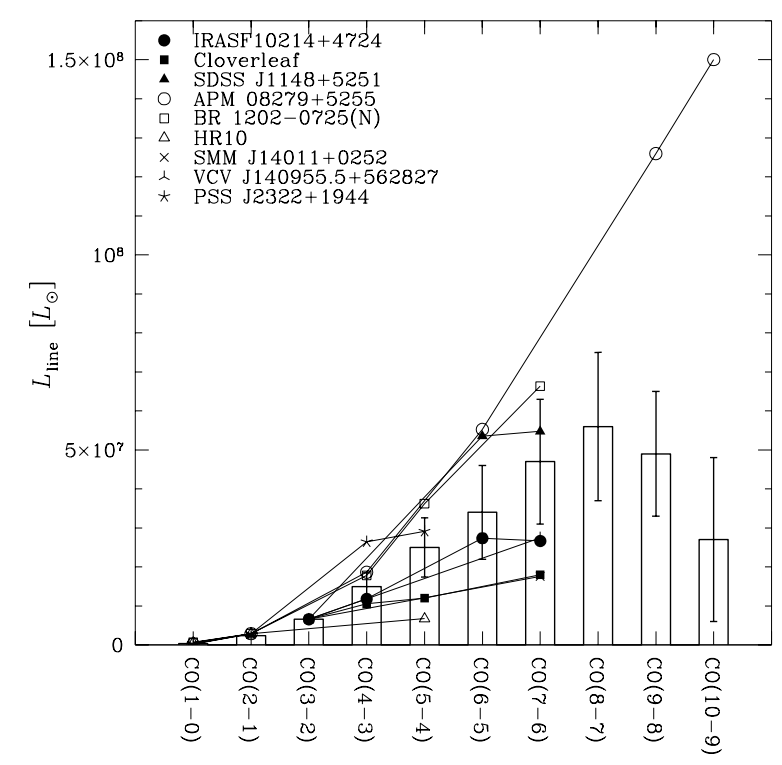

Figure 3. CO line luminosities in various high-redshift objects compared to the Mrk231 template (bar diagram). Line luminosities are normalized to the Mrk231 values at either CO $J=3-2$ or $C O J=2-1$ (Papadopoulos et al., 2007).

\section{State-OF-The ART: High- $z$ GALAXIES}

To date, detections of CO have been reported in about 50 objects at $z>1,38$ of which are mentioned in the review by Solomon \& Vanden Bout (2005). Of these 38, 22 are at redshifts $z=1.5-2.8$, the time in cosmic evolution in which both the QSO and the SMG activity peak. Multiline CO studies of the 12 brightest of these (8 QSOs and 4 SMGs with no signs of AGN activity) are being carried out using the IRAM and APEX telescopes. These obser- vations typically cover CO rotational lines from $J=3-2$ to $J=8-7$ (Weiß et al., 2005). A compilation, compared with the Mrk 231 flux distribution of Papadopoulos et al. (2007) is shown in Fig. 3.

The location of the peak of the CO flux distribution reveals different excitation conditions within the sample. For QSOs the peak typically occurs at the $J=6-5$ or $7-6$ transition, indicative of a gas density of $\sim 10^{4} \mathrm{~cm}^{-3}$ and a kinetic temperature of $\sim 50 \mathrm{~K}$. For APM $0827+5255$ the $\mathrm{CO}$ excitation is by higher by a large margin. Here the flux distribution peaks at the $J=10-9$ transition. The SMGs in the sample tend to have a lower gas excitation than the QSOs with a peak of the CO ladder typically at the $J=$ 5-4 line. These CO ladders are similar to those observed in the central region of some local starburst galaxies (e.g., M82 and NGC 253, see references in Section 3). The lowest CO excitation is observed toward the extremely red object HR 10 where the turnover of the flux distribution already occurs at the CO $J=4-3$ line (Papadopoulos \& Ivison, 2002).

Although this set of $\mathrm{CO}$ lines constrains the properties of the bulk of the CO emitting gas, surprising results are obtained when the CO ladder is probed beyond the peak, as can be seen in the two QSOs at $z \sim 4$ (APM $08279+5255$ and PSS 2322+1944) where the observations even include $\mathrm{CO}$ lines up to $J=11-10$, in the restframe $\mathrm{THz}$ regime (Weiß et al., 2007). The CO $J=9-8$ and 11-10 lines in the QSO host galaxy PSS 2322+1944 $(z=4.1)$ show a clear flux excess compared to the single excitation model which describes the peak of the CO ladder and require a second gas component with even higher $\mathrm{CO}$ excitation. This component may be related to a denser component (also indicated by HCN observations), or alternatively to a hotter component heated by the AGN such as in APM 08278+5255, and can be modeled using the CO ladders shown in Fig. 1. Interestingly, a similar CO ladder is observed towards the Galactic center, but there the high-excitation component is energetically much less important.

In summary, observations of high- $z$ sources allow access to diagnostic lines of $\mathrm{CO}$ which are very difficult to obtain from ground based observations for sources in the local universe. This leads to the somewhat paradoxical situation, that the gas properties for some high- $z$ redshift objects are currently better constrained than those of most nearby infrared-luminous galaxies. The situation will be significantly improved by the HerCULES project, referred to in Section 3. Expanding such observations to lower luminosity galaxies, larger distances and higher $J$ CO lines, in order to fully exploit the diagnostic use of the CO ladder, will be a major task for SPICA/SAFARI.

\section{5. $\mathrm{H}_{2}$ COOLING EMISSION FROM PRIMORDIAL GALAXIES}

A key question is what happens in galaxies of extremely low metallicity, where the [C II], [O I] and CO cooling chan- 


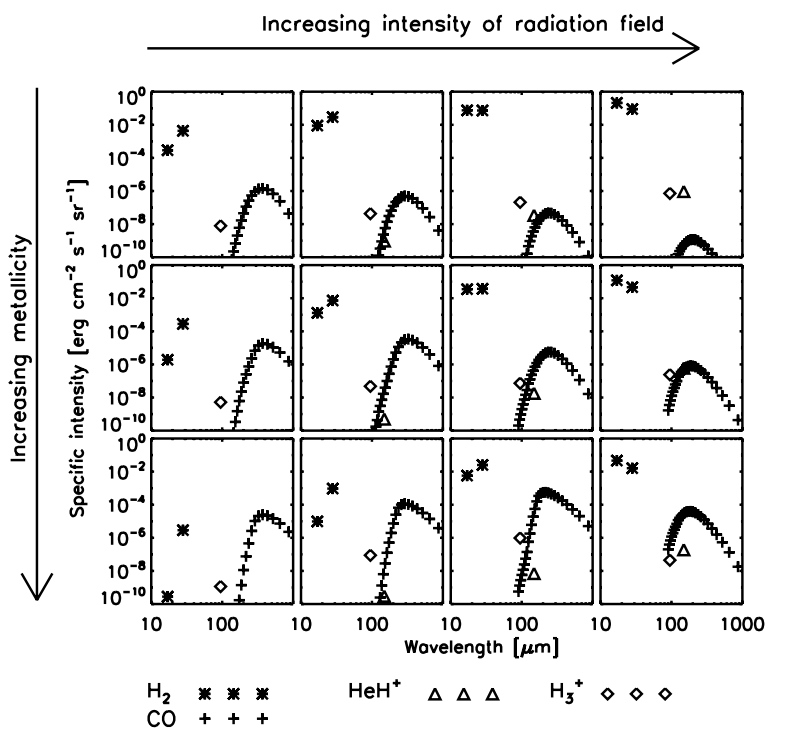

Figure 4. Rest frame spectral line distributions of high-J CO, $\mathrm{H}_{2} \mathrm{~S}(0)$ and $\mathrm{S}(1)$ (as well as lines from $\mathrm{H}_{3}^{+}$and $\mathrm{HeH}^{+}$) are shown as functions of metallicity and $X$-ray flux for a density of $10^{5} \mathrm{~cm}^{-3}$. The impinging $X$-ray flux takes on values of 0.1 , 1,10 and $100 \mathrm{erg} \mathrm{s}^{-1} \mathrm{~cm}^{-2}$; and the metallicity values of $10^{-3}$, $10^{-2}$ and $10^{-1}$ of solar (Spaans \& Meijerink, 2008).

nels are not available. In standard cosmological models, a significant population of dark matter halos collapses at redshifts larger than 5, aided by atomic (H I and He II Lyman $\alpha$ ) and molecular ( $\mathrm{H}_{2}$ and HD) cooling, and hosts the first stars and accreting black holes (Haiman et al., 2000). The $\mathrm{H}_{2}$ rotational and ro-vibrational lines are accessible to SAFARI and cool the (molecular) medium in systems with metallicities $\leq 10^{-2}-10^{-3}$ Solar. As shown by Mizusawa et al. (2005) and Spaans \& Meijerink (2008), a number of these lines are expected to be detectable with JWST, ALMA and SPICA/SAFARI, over a wide range of physical conditions.

$\mathrm{H}_{2}$ cooling emission from population III star forming regions at $z \sim 20$ will be too faint for detection, but primordial star forming gas in $z \sim 5$ galaxies should be detectable with SAFARI due to the enhanced ionization and molecule formation caused by virialization shocks (Mizusawa et al., 2005). Furthermore, the presence of an accreting black hole leads to the copious production of X-rays. In such X-ray irradiated gas, typical line intensities can be of the order of $0.1-1.0 \mathrm{erg} \mathrm{s}^{-1} \mathrm{~cm}^{-2} \mathrm{sr}^{-1}$ for the pure rotational lines of $\mathrm{H}_{2}$ at 28,17 , etc. $\mu \mathrm{m}$, which is as much as $10-30 \%$ of the black hole's accretion-driven X-ray luminosity (Spaans \& Meijerink, 2008). Flux distributions for various parameters are shown in Fig. 4, which shows the dominant role of $\mathrm{H}_{2}$ at the lowest metallicities.

Given the tight relation between supermassive black holes and the stellar spheroids that host them, it seems reasonable to use the extended Press-Schechter formalism and assume that (a) the $M_{\bullet}-\sigma$ holds at high redshift too, so the black hole mass is $2 \times 10^{-4}$ of the total dark matter mass, that (b) black holes radiate at $10 \%$ of Eddington, yielding an X-ray dominated zone of $50-400 \mathrm{pc}$, and that (c) star formation and black hole growth are roughly simultaneous, with similar contributions to the luminosity. The latter implies star formation rates of $10^{2} M_{\odot} \mathrm{yr}^{-1}$ within the central $2 \mathrm{kpc}$, quickly enriching the pristine gas, but the presence of a black hole alone is quite sufficient for SAFARI detections.

For the $\Lambda$ CDM concordance model, one then finds about 10 of such sources per $2^{\prime} \times 2^{\prime}$ field-of-view. The main contribution comes from $z<10$ halos with masses $\geq 10^{9} \mathrm{M}_{\odot}$ that yield $\mathrm{H}_{2}$ line fluxes of $\geq 10^{-18} \mathrm{~W} \mathrm{~m}^{-2}$, comfortably above the $2 \times 10^{-19} \mathrm{~W} \mathrm{~m}^{-2}$ SAFARI detection threshold. Typically, at least two pure rotational $\mathrm{H}_{2}$ lines (e.g., $\mathrm{S}(0)$, $\mathrm{S}(1), \mathrm{S}(2)$, etc.) are detectable for a $z \sim 5$ source, allowing line confusion due to redshifted mid-infrared lines from far-infrared sources to be dealt with. SPICA/SAFARI targets will likely be provided by JWST, which will discover many $z \geq 5$ galaxies.

\section{ACKNOWLEDGEMENTS}

Much of the work reported here was carried out in collaboration with Padelis Papadopoulos, Kate Isaak and Rowin Meijerink, without whose enthusiasm and perseverance these projects would have been less successful and less fun. We also thank Dominik Schleicher and Ralf Klessen for good discussions.

\section{REFERENCES}

Alexander, D., et al., 2005, Nat, 434, 738

Blain, A., et al., 1999, MNRAS, 302, 632

Bradford et al., 2003, ApJ, 586, 891

Croton, D., 2006, MNRAS, 369, 1808

Ferrarese, L., \& Merritt, D., 2000, ApJ, 539, L9

Genzel, R., et al., 2001, ApJ 563, 527

Güsten et al., 2006, A\&A, 445, L115

Haiman, Z., Spaans, M., \& Quataert, E., 2000, ApJ, 537, L4

Magorrian et al., 1998, AJ, 155, 2285

Mao et al., 2000, A\&A 358, 433

Mizusawa, H., Omukai, K., \& Nishi, R., 2005, PASJ 57, 951

Murray, N., et al., 2005, ApJ, 618, 569

Papadopoulos, P.P., \& Ivison, R.J., 2002, ApJ, 564, L9.

Papadopoulos, P.P., Isaak, K.G., \& Van der Werf, P.P., 2007, ApJ, 68, 815

Shields, G., et al., 2006, ApJ, 641, 683

Solomon P., \& Vanden Bout, P.A., 2005, ARA\&A 43, 677

Spaans, M. \& Meijerink, R., 2008, ApJ, 678, L5

Weiß et al., 2005, A\&A, 440, L45

Weiß et al., 2007, A\&A, 467, 955 